\title{
Primary Liver Lymphoma: A Case Report and Literature Review
}

\author{
Bourhafour Mouna, Allam Wafae, M'rabti Hind, Errihani Hassan
}

Department of Medical Oncology, National Institute of Oncology, Rabat, Morocco.

E-mail: bourhafourmouna@yahoo.fr

Received June 29 $9^{\text {th }}, 2011$; revised November 20 ${ }^{\text {th }}$ 2011; accepted December $6^{\text {th }}, 2011$.

\begin{abstract}
Primary non-Hodgkin lymphoma of liver is a very rare malignancy. The disease is poorly understood and few clinical studies have been conducted to help elucidate the natural course of disease, pathogenesis, optimal therapy, response to therapy, and survival. Here, we present an interesting case of primary non-Hodgkin lymphoma (NHL) originating in liver. A literature review of clinical features, diagnosis, and management is also provided.
\end{abstract}

Keywords: Non-Hodgkin Lymphoma, Liver, Hepatic Tumor, Primary Liver Lymphoma, Chemotherapy, Rituximab

\section{Introduction}

Primary liver lymphoma (PLL) is a very rare disease and is frequently overlooked as a possible diagnosis. It is defined as an extranodal lymphoma of the liver without involvement of any other organ. PLL constitutes $0.4 \%$ of cases of extranodal non-Hodgkins lymphoma, and comprises approximately $0.01 \%$ of non-Hodgkin's lymphomas [1]. The rarity of the disease causes problems in diagnosis and management. We present an interesting case of primary non-Hodgkin lymphoma originating in liver.

\section{Case Report}

A 70-year-old woman presented with a 2-month history of abdominal pain and vomiting without other gastrointestinal symptoms. His past history was relevant for ischemic cardiopathy. There was no history of alcohol intake, medications, or illicit drugs. A physical examination was unremarkable. The liver and spleen were normal in size. No superficial lymphadenopathy was present.

Laboratory results included hemoglobin $13.3 \mathrm{~g} / \mathrm{dL}$ and a white cell count of $5.5 \times 109 / \mathrm{L}$, with a normal differential. Alanine aminotransferase, aspartate aminotransferase, alkaline phosphatase (alp), and lactate dehydrogenase (ldh) were within normal limits. Levels of serum alpha-fetoprotein and carcinoembryonic antigen (cea) were not elevated. Serology was negative for HIV and for the hepatitis C (HVC) and B (HBC) viruses. Serum calcium was within normal limits.

Systematical ultrasound and computed tomography of abdomen and pelvis showed multiple hypodense nodules in the right lobe of the liver with hepatosplenomegaly without portal vein thrombosis "Figure 1". The pancreas, spleen, and biliary tract were normal. Radiography and computed tomography of chest did not reveal any mediastinal lymphadenopathy.

Histologic analysis after ultrasound-guided biopsy of the lesion showed diffuse infiltrates and small-to-intermediate atypical cells consistent with lymphoma. Immunostaining of the tumour cells showed reactivity for CD20, CD5, CD45, and Bcl6 "Figure 2". The patient was diagnosed with primary large B-cell lymphoma stage IE of liver, given that no additional foci of lymphoma were found anywhere else in the body. Chemotherapy CHOP (cyclophosphamide-doxorubicin-vincristine-prednisone) was initiated but the patient died five days after the first cycle of myocardial infarction.

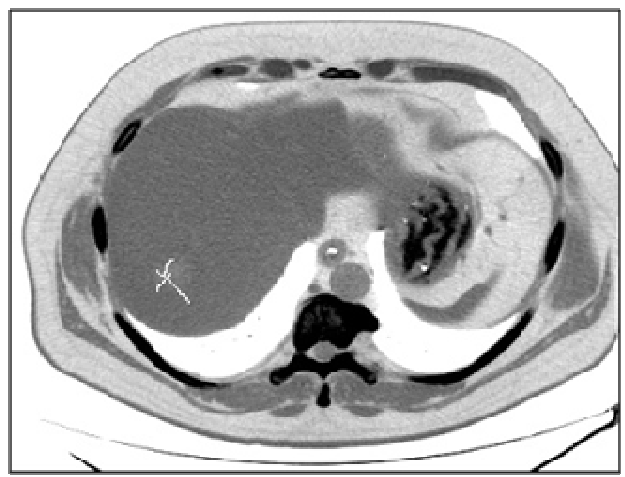

Figure 1. Computed tomography scan of abdomen, showing a hypodense nodule in the right lobe of the liver. 


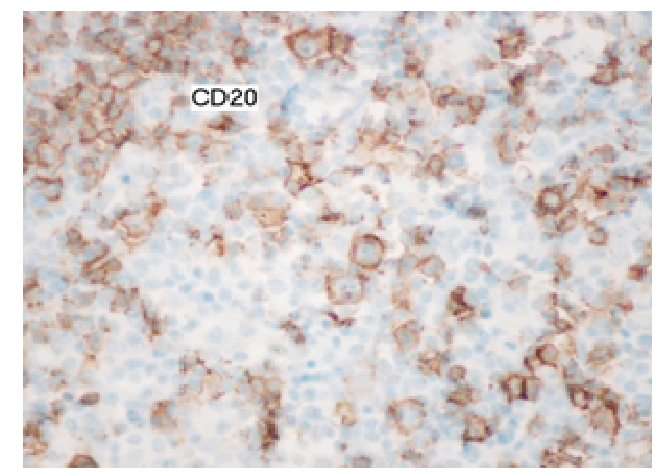

Figure 2. A photomicrograph shows tumour cells positive for CD20, proving B-cell lineage.

\section{Discussion}

Although secondary liver involvement of lymphoma in the advanced stage is common, primary liver lymphoma (PLL), which is defined as lymphoma either confined to the liver or having major liver involvement, is extremely rare [1,2]. PLL constitutes $0.4 \%$ of cases of extranodal non-Hodgkins lymphoma, and comprises approximately $0.01 \%$ of non-Hodgkin's lymphomas [1].

The exact cause of PHL is still not clear. A number of recent reports showed a higher prevalence of HVC or HBV infection in PLL patients. Hepatitis C is found in $40 \%-60 \%$ of patients with PHL; however, our patient was not positive for HCV or HBV [3]. Other reports described that it might be associated with cirrhosis and immunosuppressive drugs.

Primary hepatic lymphoma commonly presents at 55 years. There is a trend towards male predominance. Symptoms are usually non-specific. The most common presenting symptom is abdominal pain. Hepatomegaly is frequent, and jaundice is an occasional finding at physical examination $[1,4]$. The majority of cases of PHL are diffuse large B-cell lymphoma. Other histologies, including diffuse mixed small and large cell, lymphoblastic, diffuse immunoblastic, diffuse histiocytic, mantle cell, and small non-cleaved or Burkitts lymphoma, have been described in less than $5 \%$ of cases [5].

The initial radiologic test performed is usually an ultrasound; lesions are hypo-echoic relative to normal liver. On CT scans, PHL lesions appear as hypo-attenuating lesions with a central area of low intensity indicating necrosis. PLL shows hypo-intensity on T1-weighted and hyper-intensity on T2-weighted imaging in MRI [6]. The final diagnosis of PLL relies on histological examination. Liver biopsy remains the most valuable tool for diagnosis of PHL [5]. The optimal therapy is still unclear and the outcomes are uncertain. Some clinicians using surgery only, other prefer chemotherapy aloneor combined with radiotherapy [7].

Most patients are treated with chemotherapy. The standard treatment for patients with diffuse large B-cell lymphoma is CHOP. The addition of rituximab, to the CHOP regimen, when given in 8 cycles, augments the complete response rate and prolongs event-free and overall survival in elderly patients with diffuse large B-cell lymphoma, without a clinically significant increase in toxicity $[8,9]$.

\section{Conclusions}

PLL confined to the liver without lymph node or bone marrow involvement is extremely rare. If the clinical picture is suspicious for PHL, a liver biopsy should be obtained, because the disease is treatable, and with new therapeutic drugs such as rituximab, overall survival has improved for these patients. The prognosis is variable, with good response to early aggressive combination chemotherapy.

\section{REFERENCES}

[1] V. Noronha, N. Q. Shafi, J. A. Obando and S. Kummar, "Primary Non-Hodgkin's Lymphoma of the Liver," Critical Reviews in Oncology/Hematology, Vol. 53, 2005, pp. 199-207. doi:10.1016/j.critrevonc.2004.10.010

[2] A. Masood, S. Kairouz, K. H. Hudhud, A. Z. Hegazi and A. Banu, "Primary Non-Hodgkin Lymphoma of Liver," Current Oncology, Vol. 16, No. 4, 2009, pp. 74-79. doi:10.3747/co.v16i4.443

[3] F. S. Haider, R. Smith and S. Khan, "Primary Hepatic Lymphoma Presenting as Fulminant Hepatic Failure with Hyperferritinemia: A Case Report,” Journal of Medical Case Reports, Vol. 2, 2008, p. 279. doi:10.1186/1752-1947-2-279

[4] J. S. Salmon, M. A. Thompson, R. C. Arildsen and J. P. Greer, "Non-Hodgkin's Lymphoma Involving the Liver: Clinical and Therapeutic Considerations," Clinical Lymphoma, Myeloma \& Leukemia, Vol. 6, No. 4, 2006, pp. 273-280. doi:10.3816/CLM.2006.n.001

[5] R. D. Page, J. E. Romaguera, B. Osborne, et al., "Primary Hepatic Lymphoma: Favorable Outcome after Combination Chemotherapy," Cancer, Vol. 92, No. 8, 2001, pp. 2023-2029.

doi:10.1002/1097-0142(20011015)92:8<2023::AID-CNC R1540>3.0.CO;2-B

[6] K. Coenegrachts, D. Vanbeckevoort, K. Deraedt and W. Van Steenbergen, "Mri Findings in Primary Non-Hodgkin's Lymphoma of the Liver,” JBR-BTR, Vol. 88, No. 1, 2005, pp. 17-19.

[7] V. Noronha, N. Q. Shafi, J. A. Obando and S. Kummar, "Primary Non-Hodgkin's Lymphoma of the Liver," Crit Critical Reviews in Oncology/Hematology, Vol. 53, No. 3, 2005, pp. 199-207. doi:10.1016/j.critrevonc.2004.10.010

[8] M. C. Winter and B. W. Hancock, "Ten Years of Rituxi- 
mab in NHL," Expert Opinion on Drug Safety, Vol. 8, No. 2, 2009, pp. 223-235. doi:10.1517/14740330902750114

[9] R. Marcus and A. Hagenbeek, "The Therapeutic Use of
Rituximab in Non-Hodgkin's Lymphoma,” European Journal of Haematology, Vol. 77, No. 6, 2007, pp. 5-14. doi:10.1111/j.1600-0609.2006.00789.x 